\title{
Is She Mad at Me? \\ tone and conversation in text messaging
}

Kate Lucey

\section{INTRODUCTION}

The average American college student text messages constantly throughout the day. According to a Boston area Verizon retailer, it is not uncommon for young adults (an age range he did not define) to send 8,000 text messages a month. Texting has become an increasingly more important form of communication in our culture, and our country's colleges are the hotbeds of linguistic activity and change within this particular medium. Its emergence and popularity have not gone unnoticed in the sociolinguistic community, where text messaging and online conversation have become rich new areas of linguistic data yet unexplored. Most famous in this discussion is British linguist David Crystal, whose recent research, culminating in his 2008 book Txting: The Gr8 Deb8, has incited discussion and suggested fascinating conclusions about the sophistication of this means of communication and its influence on the English language.

Crystal writes a great deal about the acronyms and abbreviations within the grammar of the Short Messaging Service (SMS), but in this paper I focused on the relationship of tone and punctuation, a topic that emerged out of a conversation with a fellow student. She was struggling to compose a message to a recent acquaintance (and potential romantic interest), and could not decide on the proper end punctuation for the attitude she wanted to project. The message content, something mundane about her hometown in Maryland, wasn't the problem; it was deciding the appropriate way to finish the message off, to apply a tone. We ran through the list of options and discussed all of their implications, trying to identify which one would best convey her overall attitude: interested and engaged, but definitely light and casual. After trying a few different combinations of haha's and exclamation points we landed on something satisfactory and she sent it off.

Reflecting on it later, I realized that we had just achieved a pretty complex linguistic act. With limited resources we managed to make sure her recipient knew how she felt; her message served as a vehicle for an emotional transfer. Text messages that are pure content are all business, just an instant communication of needs to another person who could be in any place and engaged in any activity. Without explicit markers of tone, such a message is bold and possibly disconcerting, and not at all analogous to face-to-face conversation, in which politeness is paramount. American college students therefore employ punctuation and add certain particles in order to avoid ambiguity of tone and preserve standards of politeness in text messaging. 


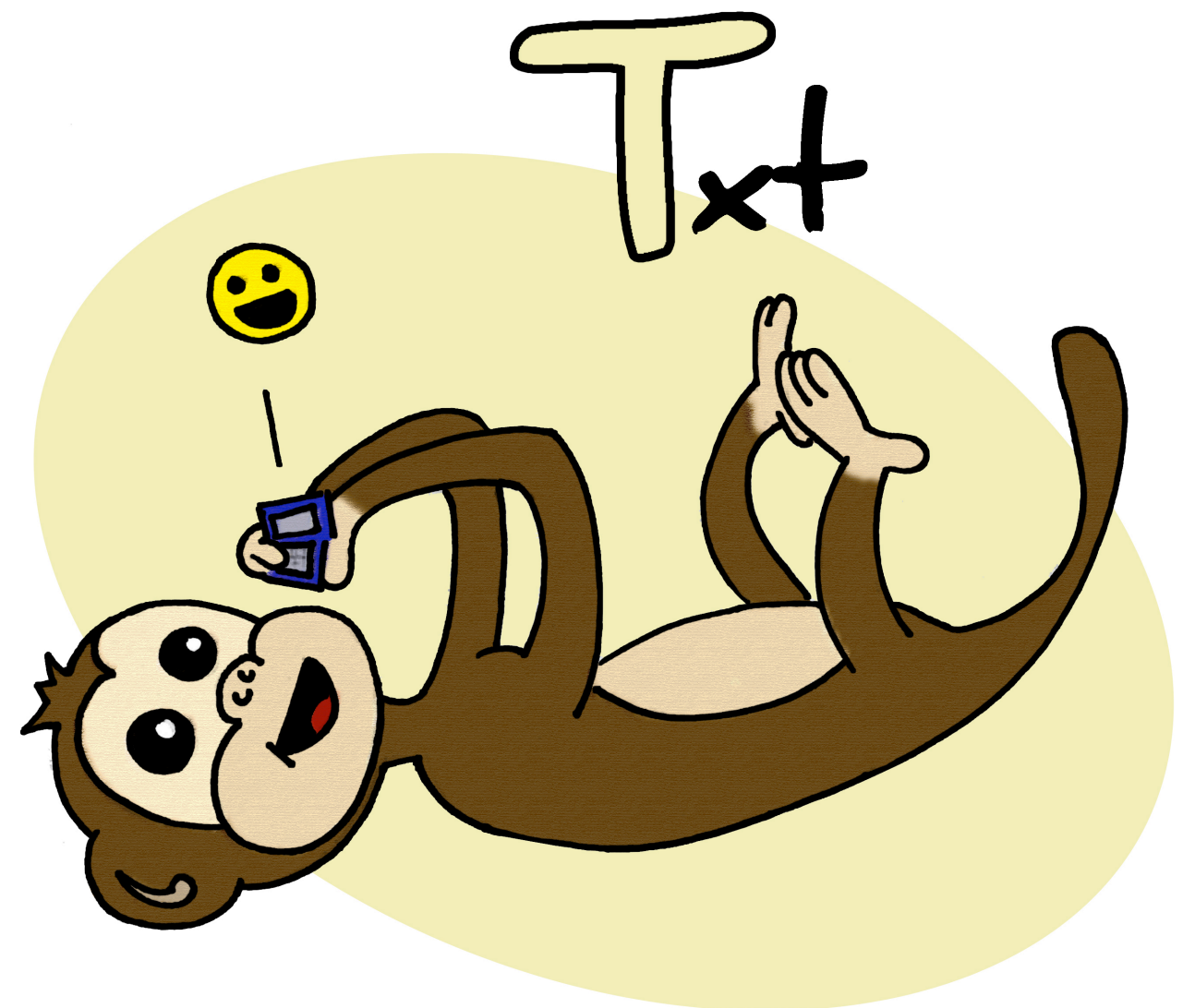




\section{Methods}

\section{a. Subjects}

There were I22 college-aged subjects who participated in this study, mostly but not limited to Boston College students. All grades were represented, although there was a high percentage of sophomores based on the data that was collected about age of participants. Only about half of the students involved responded with that information, so exact numbers are not attainable from this data set. (Appendix) ${ }^{\mathrm{I}}$

\section{b. Materials}

I conducted my work through an online survey (Appendix). I gathered the preliminary material for that survey through a Facebook page on which students were invited to post text messages of their own (sent or received) that had punctuation, or perhaps where they felt there was some ambiguity regarding punctuation. I chose to do this study in survey format for the sake of collecting large amounts of data. Presenting it this way is also beneficial because it is analogous to the medium in which text messages are normally sent and received.

\section{c. Procedure}

First I set up the Facebook page for students to post texts, and gave them about a week to do so, in which time I collected about 30 texts. I then used those sample texts as a foundation for the survey, sometimes leaving them as-is and sometimes taking the originals and changing the punctuation or use of tone words to compare the difference in interpretation. All of the examples used for analysis have therefore been created out of a sample from live subjects. In writing the survey I struggled to ensure that the questions properly identified the features I wanted to study, and that the resulting data would adequately provide evidence of what I hoped to see in a way that was statistically significant. I ran a test group and those respondents provided insight into some problems with my original questions and the overall format of my survey, which I then adopted. The test group had questions regarding the context and the role of various parties in the text exchange, which signaled to me that punctuation had not been isolated as the single variable for tone at that point. It also compelled me to provide a more equal balance of open response versus multiple choice or ranking questions, so that some of those idiosyncratic differences or comments could surface and provide color to the discussion.

I posted the survey on Facebook, making it available to all members of the Boston College community. All participants are kept anonymous, and the only personal information they were asked to provide was their university and year of graduation, which not all participants chose to do. The data I collected is therefore not linked to any particular person.

\footnotetext{
${ }^{1}$ All appendices can be found at http://goo.gl/I7KAJ.
} 


\section{RESULTS}

The data that I collected generally support my hypothesis. They show that a change in punctuation of a text message can alter its overall meaning. Furthermore, text messages without punctuation are toneambiguous, which gives readers an overall negative association to those messages. Finally, the data suggest that there are norms emerging in text message punctuation for creating explicit tone and therefore alleviating concerns of possible miscommunication.

\section{Discussion}

\section{a. Punctuation and Meaning}

Survey questions 3, I3, and I5 show the profound semantic weight of punctuation. In each of the three questions participants were asked to consider a set of texts, each with the same content but containing minor changes in punctuation. They were then asked the question "Are the authors of these texts saying the same thing?"

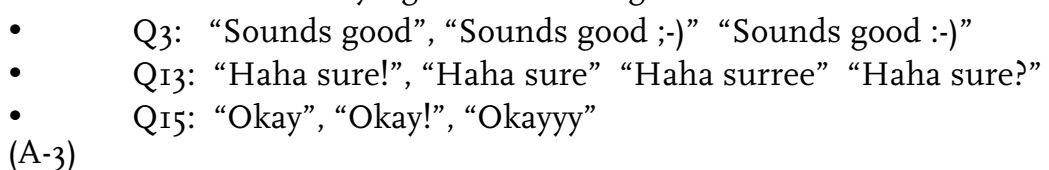

Respondents overwhelmingly answered "no," 88\%, 93\%, and 70\% respectively. When asked to explain their choices, they replied with a wealth of information concerning the wide range of meanings multiple messages with the same content words could have.

- The wink and smiley emoticons definitely mean two different things. The wink almost seems to be flirtatious. (3)

- $\quad$ Every extra letter or punctuation has a meaning (13)

- "haha sure?" indicates uncertainty and "haha surree" indicates sarcasm. The other two seem to be the same. (13)

- "okay" means they're fine with it. "okay!" means they are actually OK with the situation and happy about it. "okayy" means they are not totally satisfied with the situation. (15)

They interpreted many of the markers as signals of emphasis, as well as varying degrees of enthusiasm, and also drew conclusions about genuineness versus sarcasm, certainty versus uncertainty, comfort versus discomfort, and positivity versus neutrality and negativity. Respondents reached all of these conclusions, and yet none of the texts within a question set varied by more than two characters. This led me to conclude that there are two layers to each text message: the content, "what I am saying," and then the overlay of tone created by the non-content material such as punctuation, "what I mean by it." The words might be the same but a message with an exclamation mark performs a completely different speech act than one with an ellipsis. In face-to-face conversation a lower pitch, lack of eye contact, or a smile provides a wealth of information about the speaker's attitude. Similarly, a change in punctuation allows a sender to communicate his or her intentions with equal precision. My sample 
population of texters was highly attuned to these subtle differences and their magnitude in the text discourse.

\section{b. Lack of punctuation and ambiguity}

In these same explanations respondents not only identified the differences between different punctuation marks, but also between using punctuation and leaving the text bare, meaning that "what I mean" layer is unmarked. The discussion culminates in a general consensus that the tone of a text message with no punctuation is almost impossible to interpret, which, as the previous section demonstrates, obscures much of the meaning.

Nearly every comment concerning a "bare" text message began with an identification of neutrality of tone: "the lack of punctuation is pretty straightforward," "[it's] just neutral agreeing," "[it's] a flat statement." (A-3) Within the same comment, however, many individuals also conceded to other possible readings, such as: dismissive, indifferent, not excited, professional, serious, businesslike, negative, slightly annoyed, positive, unconvinced, or even bitchy. Evidently lack of punctuation does not imply a lack of tone, it simply creates tone ambiguity. While the participants recognized that these texts could probably be taken at face value, they are also aware of what is possibly being left unsaid, and that can be disconcerting. It leaves a lot more decision-making up to the receiver, whereas there is little to misinterpret, for example, when a visual depiction of happiness is stamped next to an affirmative statement. As one individual put it:

adding an exclamation mark or smiley face is so simple that when someone does not use one it can seem intentional.

The constrained environment of a text message magnifies the effect and increases the importance of punctuation while also making it more obligatory.

\section{c. Patterns}

College students are professional and habitual text messagers. The ability of my informants to so readily interpret and evaluate the messages I presented them reflects that, and supports the claim that there exists a set of punctuation norms within the field of texting for this speech community.

One of these norms is the "Positivity Hierarchy" in Figure I. I defined this hierarchy from compiled responses to questions 2 and 5 (A-2), which asked participants to rank the positivity of base content text messages with differing punctuation.

Figure 1:

CAPS $>(!)>[$ none $]>()>.($ ? $)>(\ldots)$

Example: HAHA > haha! > haha > haha. > haha? haha... 


\section{i. CAPS}

Used in much the same way in customary writing, this strategy of capitalization an entire word serves to intensify the sentiment being expressed. I tested for positivity, but it would follow logic for capitalization to set the extreme for other emotions.

ii. (!)

6r\% of respondents said they use CAPS when texting, compared with $79 \%$ who use exclamation points, and so it is fair to assume that for some individuals the exclamation point is the positivity hierarchy's uppermost limit. (A-I)

iii. [none] versus (.)

The meaning difference between these two markers is not fully accessible with surface level analysis, but they do have distinct roles in the hierarchy. Further investigation into this subject is required, but participants themselves raised several hypotheses on the subject as they reflected on their own use of such punctuation.

I generally don't use much punctuation, the period would show that I was a bit frustrated were I to use it, especially since the period creates a difficultto-miss syncopation in the reading of the already short text. (A-6)

In my opinion, including periods at the end of a shorter text message response indicates being stern or unhappy with the other person. (A-7)

Therefore, although the period is required in the standard grammar of written English, the unique environment of text messages sets a new standard. In such a small sample of language, a single period means much more than it would within a larger one, and so the texting standard becomes no punctuation at all. This follows the earlier analysis of texts with a lack of punctuation as being tone-ambiguous. The period is superfluous for the receiver's understanding of content, and so adding it conveys tone. The subjects hint and I would hypothesize that standard punctuation is used in longer text messages and not seen as carrying the negative tone described above because it serves its normal function of providing clarity and distinguishing between sentences.

iv. (?)

The standard usage of a question mark is to represent the upward pitch contour present in spoken questions. This function is exhibited in text messages in true questions, but also on statements. The projection of a question-type inflection on a declarative can signal several things. My respondents reported:

- If they respond with a question mark then it definitely puts doubt in my mind that they are comfortable.

- [in] the response to the question mark the person could be uncomfortable or confused

- $\quad$ the question mark one is like a tentative agreement like they actually aren't quite sure how to answer 
- the question mark shows some sort of questioning like an "I guess so" (all A-3.c)

To generalize, the question mark takes the overall power of an assertion and then diminishes or qualifies it to reflect something less confident or less positive in the sender's attitude, something along the lines of "I'm going to say this, but I want you to know it's dubious."

v. $(\ldots)$

The ellipsis, true to standard grammar, is an incompletion of a thought, which my respondents reported was a sign of uncertainty or discomfort, and highly negative. (A-3.C)

I constructed the hierarchy based on the responses to questions 2 and 5 (A-2), which asked explicitly for ranking, but significantly the ordering was also maintained in the responses to question $6(\mathrm{~A}-2)$. The participants were not asked to rank the punctuation but instead mark any text messages that might make them feel uncomfortable if they were to receive it in response to a request. Interpreting the feeling of discomfort as a lack of positivity, the hierarchy is maintained. Isolating the examples in which the content word is 'sure,' the data was this:

Figure 2:

\begin{tabular}{|c|c|}
\hline Text & \% of participants uncomfortable \\
\hline sure... & 76 \\
\hline sure. & 61 \\
\hline sure & 56 \\
\hline sure! & 23 \\
\hline Sure haha & 27 \\
\hline
\end{tabular}

$92 \%$ of the students who took my survey reported that they use the particle "haha" in their own text messages, making it the most popular of the options. (The questions mark was second with $83 \%$ reported usage) (A-I). It can be considered to be synonymous with "lol", an acronym for "laughing out loud," that only garnered 35\% reported usage from my respondents. Outside of the realm of punctuation, but not quite words in the traditional sense, the best way to describe them is as onomatopoeia for laughter, and they definitely have weighty significance concerning tone. This does not mean, however, that they are only used when the sender is actually laughing, thinks something is funny, or even always mark positive tone. Question 7, below, displays exactly this point.

Question 7: Choose the best option for each. The author of this text is:

\begin{tabular}{|l|l|l|l|l|}
\hline Text/Adjective & Laughing & Uncomfortable & Sarcastic & Other \\
\hline A & 16 & 66 & 11 & 7 \\
\hline B & 81 & 3 & 3 & 5 \\
\hline C & 38 & 25 & 0 & 32 \\
\hline D & 18 & 2 & 76 & 7 \\
\hline E & 13 & 28 & 0 & 56 \\
\hline
\end{tabular}

Numbers indicate percentage.

A: I mean it's your decision...haha

B: HAHA you know they were laughing when they wrote that

C: It's all good haha 
D: I hope you are having a wonderful car ride back lol

E: Hey love. I have two questions for you at some point. Nothing crazy though lol so don't worry!

It is interesting that text $\mathrm{B}$, the only one that indicates true laughter, is marked with the all-caps positivity marker, perhaps because the sender wanted to make it obvious that he or she really was laughing and not using any of the myriad of other possible tones that "haha" can convey. In a sense it has become almost like another content word in this text message, and follows the schema of positivity accordingly.

There is nothing within the content of D specifically that could point to the tone of sarcasm. "lol" itself does not have intrinsically anything that makes it sarcastic, and yet the two put together were clearly suggestive of that tone to most of the respondents. The context of this text would make it obvious whether or not this person was serious, but that the majority of those taking the survey, who had no knowledge of the situation, assumed it was sarcastic indicates that sarcasm is regularly associated with these particles, no explanation necessary.

Texts A, C, and E represent a highly common and complex use of "haha," that was further studied in questions 8 and I7 (A-5). Question 8 asks participants to supply their own adjectives when they did not feel the author's attitude was fully represented in the ones suggested by question 7 . Question I7 asks for further analysis of sentence E. In the responses to question 8, the participants, extremely capable at tackling all of the other issues presented in the survey, could not decide on how to name the way that it is used, though they could go into great detail describing what it accomplishes as a part of a speech act. They described it as a "flavor particle" and "filler," as well as a way to make it "so that the text is taken in a less serious manner." "haha" seems to be added to texts for the sake of the reader, and the flow of the exchange as a whole.

Question I7:

Consider this text message: [HEY LOVE.] I HAVE TWO QUESTIONS FOR YOU AT SOME POINT. [NOTHING CRAZY THOUGH LOL SO DON'T WORRY!] Comment on the possible functions of the bracketed text.

Many of the responses show that the participant identified the unbracketed text as what I have been calling the content message, saying things like:

- If you just got the middle part, you might start wondering if something was wrong or your friend was mad about something they couldn't talk to you about via text.

- $\quad[$ the bracketed text] makes the "I have two questions for you at some point" much less intimidating and puts the reader at ease about what the questions are about.

The situation the question provides is a unique one, but the concerns are not. The average texter, it appears, will do what it takes to make sure he or she is not causing anxiety in their interlocutor. Particles like "haha" and even greetings like "hey love" are there to assuage any fears and disseminate any negativity that could be inadvertently present in their text message and overtly communicate a casual and comforting tone. 
It effectually shoves the tone of the text into the higher half of the positivity hierarchy no matter what the context or content of the text, as if the reader will feel more at ease just by seeing it there in the midst of the rest of the information. I see it as a signal that engaging in text messaging, especially as prolifically as college students do, is a very complex and risky linguistic act. "haha" is like a check-in from one conversation partner to another: "our relationship is still intact and I am in this with you." This is something that is so easy to convey with a smile, with body language, with a really peppy tone of voice, but is not so simple in text messaging.

By nature, text messages are instant. That is the point, actually. People can have entire conversations in a matter of minutes, and accomplish a great deal of business that way. This is not how normal conversation goes, however, much of the work in a face-to-face conversation is doing the dance of politeness, of ensuring that neither person crosses the line and causes harm to that person's overall image. When that person is not in front of you, however, how can you judge your success? Or worse, how do you measure the possible damage? What the survey responses tell me is that this is something that college students are intensely aware of as they have text exchanges. Since they are not directly connected to their conversation partner and cannot engage in the normal conversational work of greetings, politeness features, back channeling, nodding, smiling, and all of the rest of what encourages and reassures us throughout our conversations, they are incredibly precise and perhaps overly generous with their tone cues. This is again why a text that is all "this is what I'm saying" and not "this is what I mean by it" is at high risk for being rude or off-putting, for causing its receiver the discomfort of wondering "are we okay?"

\section{CONCLUSION}

Although my study was successful, there are things I would change. Questions 9-I2 did not providing the answers I had intended with regards to the effect and impact of "haha" as a text-symbol, something that I attribute to poor question asking on my part (A-6). Secondly, I struggled to isolate a variable without giving total transparency of my hypothesis throughout the creation of the survey. Finally, there were some technical issues with the survey software, in which respondents were not able to go back and review past questions when they related to each other, which most likely meant a loss of possible data as well as a decrease in its viability.

The field of text messaging is saturated with fascinating research questions with important implications concerning language and social interaction. For further study I would like to analyze the effect of gemination of letters within words as a means of tone, something that was displayed in my survey as being a legitimate strategy, but that could not be included in the discussion at this time. The differences between the genders in style and interpretation of texting would undoubtedly produce interesting results. I would like to go back and re-ask some of my questions with different sample texts and different combinations of punctuation, including all five markers this time, to continue to validate the positivity hierarchy. I wonder if I asked the same questions concerning negativity or overall intensity of emotion if the order would be the same. I think it would also be fruitful to examine the way that individuals' styles change depending on their relationship with their interlocutor. This was 
something that was alluded to on various occasions in my data, and that I did not have the means to fully explore.

If nothing else, what I have shown is that text message punctuation has a highly communicative function, and an incredibly important one within each text exchange. Furthermore, I have shown that the standards of politeness and clarity present in face-to-face interactions are still paramount in these brief I6o-character bits of language, and that these standards motivate many of the specialized characteristics and linguistic features that make text messaging such a unique area of language. They may even take on a heightened significance due to the restricted environment of a text message while also becoming even more specific and regulated than in more free-form face-to-face interactions. Finally, texting is not just a reduction of "proper English," but rather there are rules forming for "good" texting, and following them is crucial to communicative success. It is important to note that clear communication and understanding of the rules is an issue recognized by frequent texters, all of whom had ready opinions and strong feelings on the subject. Their ability to assess and critique strangers' text messages and describe their linguistic features, and their understanding that "every extra letter or punctuation has meaning" only emphasizes more what an artful feat it truly is to employ this method of communication.

\section{WORKS CITED}

Crystal, David. (2008). Txting: the gr8 db8. Oxford: Oxford University Press.

Wardhaugh, Ronald. (2010). An introduction to sociolinguistics. Malden, MA: WileyBlackwell. 